\title{
TAXAS DE CRUZAMENTO EM UMA POPULAÇÃO NATURAL DE CRYPTOCARYA MOSCHATA NEES (LAURACEAE)
}

\author{
Pedro Luís Rodrigues de Moraes ${ }^{1,2}$ \& Reinaldo Monteiro ${ }^{3}$ \\ Biota Neotropica v2 (n2) - http://www.biotaneotropica.org.br/v2n2/pt/abstract?article+BN01102022002 \\ Recebido em 12/04/2002 \\ Revisado em 09/07/2002 \\ Publicado em 07/08/2002 \\ ${ }^{1}$ Bolsa CAPES, parte da tese de Doutoramento \\ ${ }^{2}$ Laboratório de Melhoramento de Plantas, CENA-USP, Caixa Postal 96, 13400-970, Piracicaba, SP, Brasil; Autor para \\ correspondência: E-mail: plmoraes@cena.usp.br \\ ${ }^{3}$ Departamento de Botânica, IB, UNESP, Caixa Postal 199, 13506-900, Rio Claro, SP, Brasil
}

\begin{abstract}
Outcrossing rates of a natural population of Cryptocarya moschata Nees (Lauraceae)]. The mating system of the canopy Brazilian Atlantic rain forest tree Cryptocarya moschata was studied at Parque Estadual Carlos Botelho, São Miguel Arcanjo, São Paulo, Brazil. Outcrossing rates were determined through electrophoretic allozyme markers from seedlings germinated of seed cohorts collected from 35 trees. An indirect estimate of the outcrossing rate at equilibrium gave a mean of $\hat{t}_{\mathrm{eq}}=0.51$. Direct single locus and multilocus outcrossing rate estimates were $\hat{t}_{\mathrm{S}}=0.725 \pm 0.041$ and $\hat{t}_{\mathrm{m}}=0.884 \pm 0.034$, respectively, indicating a predominant outcrossing mating system. Individual trees outcrossing rates ranged from 27 to $100(\overline{\mathrm{x}}=87.8)$ percent, from $\hat{t}_{\mathrm{m}}$ calculated by holding the population pollen allele frequency constant for each family. From Ritland's “sibling-pair" model (correlated mating model), correlation of selfing ( $\left.\hat{r}_{\mathrm{S}}\right)$ and correlation of outcrossing paternity ( $\hat{r}_{\mathrm{p}}$ ) were $35.7 \%$ and $99.0 \%$, respectively. These results corroborate the fact that there is variation in selfing rates among different trees, but it may also have indicated that when there is inbreeding, most seeds in the trees are likely to be full-sibs.
\end{abstract}

Key words - allozymes, Lauraceae, mating system, Neotropics, Cryptocarya, Atlantic rain forest, Brazil

Resumo - [Taxas de cruzamento em uma população natural de Cryptocarya moschata Nees (Lauraceae)]. O sistema de cruzamento da espécie arbórea de dossel da Mata Atlântica brasileira, Cryptocarya moschata, foi estudado a partir de material proveniente do Parque Estadual Carlos Botelho, São Miguel Arcanjo, São Paulo, Brasil. As taxas de cruzamento foram determinadas através de marcadores alozímicos obtidos de plântulas germinadas de coortes de sementes coletadas de 35 árvores. O valor médio da taxa de cruzamento de equilíbrio (estimador indireto) foi $\hat{t}_{\mathrm{eq}}=0,51$. As estimativas das taxas de cruzamento uniloco e multilocos (estimadores diretos) foram $\hat{t}_{\mathrm{S}}=0,725 \pm 0,041 \mathrm{e} \hat{t}_{\mathrm{m}}=0,884 \pm 0,034$, respectivamente, indicando um sistema de cruzamento predominantemente alogâmico. As taxas de cruzamento de árvores individuais variaram de 27 a $100(\overline{\mathrm{X}}=87,8)$ porcento, a partir de $\hat{t}_{\mathrm{m}}$ calculado com as freqüências gênicas de pólen mantidas constantes ao nível populacional. A partir do modelo de "par de irmãos" (modelo de cruzamento correlacionado) de Ritland, a correlação entre duas progênies irmãs oriundas de autofecundação $\left(\hat{r}_{S}\right)$ e a correlação entre duas progênies irmãs oriundas de paternidade por exocruzamento ( $\hat{r}_{\mathrm{p}}$ ) foram 35,7\% e 99,0\%, respectivamente. Esses resultados corroboram o fato de haver variação nas taxas de autocruzamento entre as diferentes árvores, podendo também indicar que quando há endogamia, a maior parte das sementes nas árvores são provavelmente irmãs-germanas.

Palavras-chave: alozimas, Lauraceae, sistema de cruzamento, Neotrópico, Cryptocarya, Mata Atlântica, Brasil 


\section{Introdução}

O tipo de sistema reprodutivo determina o modo de transmissão de genes de uma geração à próxima (Brown 1989) o que faz, conseqüentemente, com que as investigações sobre a dinâmica da mudança genética estejam concernidas, direta ou indiretamente, com o processo de cruzamento (Clegg 1980). Dessa forma, o sistema reprodutivo não somente determina as freqüências genotípicas em gerações subseqüentes, mas também afeta os parâmetros genéticos populacionais, tais como, tamanho de vizinhança, fluxo gênico e seleção (Hamrick 1990).

Os métodos tradicionais para a avaliação do sistema reprodutivo de uma espécie baseiam-se na observação de cruzamentos, do comportamento de agentes polinizadores, no exame da morfologia floral e de resultados de experimentos controlados de polinização. Esses métodos podem prover indicações sobre o sistema reprodutivo de uma espécie, mas não permitem uma medida direta do sucesso reprodutivo de populações, sendo inadequados para a análise ao nível populacional (Paiva et al. 1993, 1994). Tradicionalmente, as taxas de cruzamento foram investigadas pela análise de progênies de plantas possuindo marcadores morfológicos conhecidos. Pela substituição dos marcadores morfológicos pelos marcadores bioquímicos, na forma de isoenzimas, as taxas de cruzamento puderam ser estimadas mais efetivamente do que pelos métodos tradicionais (Bawa 1976).

A determinação das taxas de cruzamento em populações naturais foi sempre limitada por vários fatores, tais como: distância irregular entre plantas, especialmente em espécies arbóreas, barreiras naturais entre plantas, a dificuldade de se identificar um bom marcador genético, e a expressão de dominância exibida, na maior parte dos casos, por esses marcadores (Paiva et al. 1993, 1994). Contudo, Ritland \& Jain (1981) enfatizaram o poder e a versatilidade das estimativas quando dados eletroforéticos são empregados, considerando-se que muitos locos segregantes com alelos co-dominantes podem ser, freqüentemente, encontrados nas populações.

Os estudos quantitativos dos sistemas reprodutivos de espécies arbóreas tropicais estão ainda em consolidação (Murawski et al. 1994), no entanto, as análises isoenzimáticas de progênies têm demonstrado que os sistemas reprodutivos de árvores tropicais abrangem desde uma mistura de exocruzamento e autofecundação até o exocruzamento predominante ou completo (O'Malley \& Bawa 1987, O’Malley et al. 1988, Murawski et al. 1990, Murawski \& Hamrick 1991, 1992a, 1992b, Murawski et al. 1994, Murawski \& Bawa 1994, Hall et al. 1994, Stacy et al. 1996, Rocha \& Lobo 1998).

Segundo Hamrick \& Schnabel (1985), o procedimento mais comumente utilizado para se estimar as proporções de endogamia e alogamia em populações de plantas foi o modelo de cruzamento misto (Allard \& Workman 1963, Brown \& Allard 1970). Em espécies de cruzamento misto, as proporções de alogamia e endogamia determinariam a estrutura genética de suas populações, que se assemelhariam à estrutura genética de alógamas. Contudo, uma vez que as taxas de cruzamento poderiam variar no tempo, devido a fatores como densidade populacional ou comportamento de polinizador, gerações ou classes de idade de espécies de cruzamento misto poderiam ser geneticamente diferenciadas (Loveless \& Hamrick 1984).

Dentro do grupo de espécies arbóreas hermafroditas, vários levantamentos têm mostrado que uma grande proporção é composta por espécies autoincompatíveis (Bawa 1974, Zapata \& Kalin-Arroyo 1978, Bullock 1985, Bawa et al. 1985), que aliada à porção de espécies dióicas parecem indicar que o sistema reprodutivo predominante em espécies arbóreas tropicais é o de exocruzamento. No entanto, a maioria das espécies que foram incluídas nesses levantamentos é composta por aquelas que ocorrem em altas densidades (Bawa et al. 1985). A partir disso, Kageyama (1990) levantou a possibilidade de que a freqüência de autocompatibilidade e também de taxas de auto-polinização podem ser maiores em espécies que são encontradas em baixas densidades.

O sistema de cruzamento de espécies hermafroditas, em particular, é determinado tanto por fatores genéticos inerentes (p.ex., grau de autocompatibilidade) e por fatores ecológicos (p.ex., movimentação de polinizadores, fenologia do florescimento, densidade populacional), como apontado por Murawski \& Hamrick (1991).

De acordo com Moraes et al. (1999), a espécie Cryptocarya moschata apresenta flores hermafroditas pequenas, tendo como prováveis polinizadores pequenos insetos, como moscas, pequenas abelhas, trips. Adicionalmente, a presença de plântulas albinas e cloróticas encontradas na população (P.L.R. Moraes, dados não publicados) permite se inferir a ocorrência de endogamia, com possibilidade de autofecundação (vide O'Malley \& Bawa 1987), o que é corroborado pela presença de árvores isoladas reprodutivas. Ainda, a estimativa de taxas de cruzamento em plantas é desejável porque o sistema de reprodução tem sido indicado como o principal fator a influenciar a estrutura genética populacional (Hamrick \& Godt 1990). Com isso, o objetivo específico deste trabalho é o de analisar o sistema de reprodução de Cryptocarya moschata através da quantificação de suas taxas de cruzamento aparente e multilocos, cujo modelo é o de cruzamento misto. 


\section{Material e Métodos}

O material utilizado neste trabalho constou de folhas, tanto de plântulas como de árvores adultas de C. moschata, cuja coleta foi efetuada em plantas individuais, no Parque Estadual Carlos Botelho (PECB; 24044' a 24015'S, 47046' a $\left.48^{\circ} 10^{\prime} \mathrm{W}\right)$, São Miguel Aracanjo, SP.

Para a análise das progênies, coletou-se um dos eófilos do primeiro par emitido pelas plântulas com idade de aproximadamente 10 meses após a semeadura. Os eófilos coletados foram imediatamente submetidos à extração de isoenzimas e analisados imediata e subseqüentemente através de eletroforese horizontal em gel de amido, conforme descrito em Moraes et al. (1999, 2002).

As árvores adultas maternas tiveram a coleta de pelo menos um ramo foliar portador de folhas maduras, sendo acondicionadas em sacos pláticos, que por sua vez foram armazenados em caixas de isopor com gelo para o transporte até o laboratório. Uma vez no laboratório, foram mantidos em geladeira $\left(\cong 5^{\circ} \mathrm{C}\right)$ até sua extração e análise eletroforética após aproximadamente dois dias em média.

Foram amostradas 141 árvores adultas, distribuídas ao acaso, englobando indivíduos tanto agrupados como isolados, ao longo do sistema de trilhas disponíveis em uma área de aproximadamente 647 ha. Destas, 86 árvores tiveram 6.034 sementes coletadas e que foram germinadas em canteiros de areia, para a obtenção de plântulas, sendo que houve a germinação de 1.174 sementes. No entanto, apenas 35 árvores apresentaram um número igual ou superior a 20 progênies germinadas (= 35 famílias), que foi o número mínimo estabelecido para a inclusão nas análises. Todas as 35 árvores apresentaram-se agrupadas, ou com pelo menos um indivíduo co-específico distanciado não mais que 50 metros das mesmas. Adicionalmente, para fins comparativos e de discussão, os resultados referentes a três amostragens de populações de indivíduos juvenis (com seis anos de idade e 20 indivíduos em cada uma) de C. moschata, apresentados em Moraes et al. (2002), também estarão sendo aqui abordados.

A partir do emprego de marcadores alozímicos, revelados pela eletroforese em gel de amido, realizou-se a caracterização genética das populações analisadas, seguindo-se as recomendações de Kephart (1990) e Alfenas et al. (1991), conforme apresentado em Moraes et al. (1999, 2002). Foram utilizados 16 locos polimórficos de cinco sistemas isoenzimáticos, a saber: fosfatase alcalina (Alp-1, Alp-3, Alp-4, Alp-5), catalase (Cat-1, Cat-2, Cat-3, Cat-4), glutamato-oxaloacetato transaminase (Got-1, Got-2), peroxidase (Per-1, Per-2, Per-3, Per-4, Per-5), e polifenoloxidase (Ppo-4).

A taxa de cruzamento aparente, ou de equilíbrio (estimativa indireta), foi obtida a partir do método dos momentos, através da estimativa do coeficiente de endogamia (índice de fixação; resultados apresentados em Moraes et al. 2002), estimado diretamente para as progênies. Empregou-se a relação proposta por Wright (1921) e Nei \& Syakudo (1958) entre a taxa de cruzamento e a endogamia: $\hat{F}=\left(1-\hat{t}_{\text {eq }}\right) /\left(1+\hat{t}_{\text {eq }}\right)$, ou $\hat{t}_{\text {eq }}=(1-\hat{F}) /(1+\hat{F})$. Essa fórmula assume que a única causa de endogamia na população é devida à autopolinização e que as populações alcançaram valores de equilíbrio para $F$.

Fez-se, também, a estimativa multilocos da taxa de cruzamento $\left(\hat{t}_{\mathrm{m}}\right)$, que é uma estimativa direta, obtida a partir do modelo de cruzamento misto proposto por Ritland \& Jain (1981), através dos métodos "Expectation Maximization" (EM) e "Newton-Raphson" (NR), para a maximização da equação de verossimilhança, empregando-se os programas MLT (Ritland 1990) e MLTR v.1.1 (K. Ritland, não publicado). Obtiveram-se, também, a estimativa da taxa média de cruzamento unilocos populacional $\left(\hat{t}_{\mathrm{S}}\right)$ e o coeficiente médio de endogamia das árvores maternas $(\hat{F})$. O coeficiente médio de endogamia das progênies foi obtido através da relação $\hat{F}_{p}=\left(1-\hat{t}_{m}\right) /\left(1+\hat{t}_{m}\right)$, sendo comparado ao $\hat{F}$ das árvores maternas. As estimativas das freqüências alélicas de pólen ( $\hat{p}$ ) e de óvulo ( $\hat{o}$ ) foram feitas pelo método EM. A adequação das estimativas ao modelo de cruzamento misto foi analisada através de um $\chi^{2}$ de contingência (ElKassaby \& Ritland 1987), visto que o modelo de Ritland \& Jain está baseado nas seguintes pressuposições: 1) todos os genótipos maternos têm uma mesma probabilidade de cruzamento a partir de um conjunto polínico homogêneo; 2) os alelos de locos diferentes segregam independentemente; 3) cada alelo está em Equilíbrio de Hardy-Weinberg; 4) os marcadores genéticos não são afetados por seleção ou mutação durante o tempo de reprodução e de amostragem das progênies.

A taxa média de cruzamento unilocos foi calculada através de uma média de variância mínima dos valores de $\hat{t}_{s}$ (unilocos) de cada loco. Essa taxa é calculada para ser comparada com a taxa multilocos da população $\left(\hat{t}_{m}\right)$, pois, quando ocorre endogamia devida ao cruzamento entre indivíduos aparentados e/ou autofecundação, as estimativas multilocos de $t$ são esperadas ser maiores do que as estimativas unilocos (Shaw \& Allard 1981).

As diferenças entre freqüências alélicas de pólen e de óvulo que contribuem ao conjunto gênico das sementes ou plântulas das diferentes árvores maternas foram testadas através da estatística $\chi^{2}$, onde $\chi^{2}=N_{F_{\mathrm{ST}}}(k-1) \operatorname{com}(k-$ 1) graus de liberdade, onde $N$ é o número de gametas nos dois grupos (pólen e óvulo), $k$ é o número de alelos para o 
loco, e $F_{\mathrm{ST}}$ é a medida de diversidade genética entre populações, tratando pólen e óvulo como populações separadas (Murawski \& Hamrick 1992a, Murawski et al. 1994). As freqüências alélicas de pólen foram obtidas assumindo-se segregação Mendeliana normal dos alelos maternos. Dessa forma, a contribuição materna de alelos nas progênies foi subtraída dos genótipos das progênies para a obtenção das freqüências alélicas de pólen (Murawski \& Hamrick 1992a).

Adicionalmente, a partir do programa MLTR, estimaram-se os parâmetros correlacionados ao sistema reprodutivo, $r_{\mathrm{s}}$ e $r_{\mathrm{p}}$, conforme Ritland (1989). Esse autor apresentou um modelo que denominou de "par de irmãos", com uma abordagem paramétrica e que se baseou nas probabilidades de pares de irmãos para descrever e estimar as correlações entre irmãos oriundos de autofecundação e de exocruzamento. Esse modelo assentou-se no fato de que pela amostragem de duas progênies oriundas de uma mesma mãe, em uma população parcialmente endogâmica, estas poderiam ser ambas autofecundadas, uma autofecundada e outra exocruzada, ou ambas exocruzadas. Em relação a essas três classes, poderia haver um excesso de pares de irmãos, ambos por autofecundação, em relação às expectativas devidas ao acaso, ou uma correlação entre os dois irmãos oriundos de autofecundação. Além disso, se cruzamentos sucessivos não fossem aleatórios, os pares de irmãos, ambos por exocruzamento, poderiam ser divididos entre aqueles que compartilhassem o mesmo parental masculino versus aqueles com diferentes parentais (Ritland 1989). Desta forma, o parâmetro denominado $r_{\mathrm{s}}$, seria a correlação entre dois irmãos oriundos de autofecundação (o que seria dado pela covariância de autofecundação dividida por $s[1-s]$, onde $s$ seria a taxa de autofecundação), indicando a correlação de autocruzamento dentro das progênies. Por sua vez, o parâmetro denominado $r$ seria a correlação entre dois irmãos com paternidade devida a exocruzamento, ou a proporção de irmãos germanos entre os irmãos exocruzados.

O parâmetro de correlação a partir de paternidade oriunda de cruzamento, $r_{\mathrm{p}}$ (a correlação entre genótipos paternos), foi estimado a partir de $f$ (correlação entre gametas paternos), da seguinte maneira: $\hat{r}_{p}=[2 /(1+\hat{F})] \hat{f}$, onde $F$ era o coeficiente de endogamia dos parentais masculinos. Assumiu-se que o $F$ paterno era igual ao $F$ materno, o que foi uma pressuposição mais adequada para as espécies hermafroditas do que para as dióicas (Ritland 1989).

Em todas as análises realizadas, as variâncias das estimativas foram obtidas empregando-se 1.000 "bootstraps", onde a unidade de reamostragem foi obtida entre as famílias, no caso de estimativa populacional, ou de indivíduos dentro das famílias, no caso de estimativa para famílias individuais.

\section{Resultados e Discussão}

A endogamia existente em uma espécie apresenta-se intimamente relacionada ao sistema reprodutivo da mesma. Dessa forma, a taxa de cruzamento, ou percentual da alogamia, pode ser estimada a partir dos níveis de endogamia existentes. Nesse sentido, um primeiro aspecto a ser observado é a existência, ou não, de desvios do equilíbrio de panmixia, estimando-se posteriormente a taxa de cruzamento.

Moraes et al. (2002) testaram a aderência das freqüências genotípicas ao equilíbrio de panmixia, para o presente conjunto de dados das 35 famílias de plântulas, e encontraram que todos os locos analisados apresentavamse em desequilíbrio de Hardy-Weinberg. Para a população de árvores adultas maternas, oito dos 16 locos apresentaramse em desequilíbrio, sendo que as sub-populações de indivíduos juvenis apresentaram-se com três a quatro locos em desequilíbrio.

A taxa de cruzamento aparente $\left(\hat{t}_{\mathrm{eq}}\right)$, obtida a partir do índice de fixação ( $\hat{F}_{\text {(IS) }}=0,326$; Moraes et al. 2002) das progênies, foi de 0,51 , tomando-se todas as famílias conjuntamente como uma única "população", indicando a existência de um componente significativo de endogamia. Por outro lado, o valor obtido da taxa de cruzamento aparente para a população de indivíduos adultos foi de $0,96(\hat{F}($ IS $)=$ 0,018; Moraes et al. 2002), indicando que a espécie é preponderantemente alógama. As três sub-populações de indivíduos juvenis do PECB apresentaram índices de fixação de $0,085,-0,150$ e -0,067 (Moraes et al. 2002), resultando em taxas de cruzamento aparente de $0,84,1,35$ e 1,14, respectivamente, com um valor médio de 1,11 , indicando alogamia completa. Os valores obtidos acima de 1,0, que são "biologicamente inaceitáveis" (Cheliak et al. 1983), não são devidos a efeitos de Wahlund, ou necessariamente pela invalidação do modelo de cruzamento misto. Duas causas principais podem ser responsáveis: 1) efeitos de amostragem dentro do contexto de um modelo de cruzamento misto; e 2) cruzamentos não preferenciais (Brown et al. 1985).

A diferença considerável entre as taxas de cruzamento aparente de progênies $(51 \%)$, juvenis $(\approx 100 \%)$ e adultos $(96 \%)$ pode ser causada por dois fatores. O primeiro devido a diferenças encontradas em diferentes anos. Sob essa hipótese, o fato de que as progênies apresentaram a menor taxa de cruzamento poderia ser uma mera coincidência. $\mathrm{O}$ segundo fator poderia ser uma vantagem diferencial de indivíduos oriundos de sementes autofecundadas $v s$. sementes de exocruzamento. $O$ presente trabalho não discriminou esses fatores. Contudo, a segunda hipótese de taxa de cruzamento constante, mas com uma maior vantagem 
de indivíduos originados de sementes exocruzadas, requer que essa vantagem de viabilidade seja relativamente grande (Moran \& Brown 1980). Se isso for verdade, para a situação do presente trabalho, as diferenças encontradas para as taxas de cruzamento entre as diferentes classes de idade podem ser resultantes de seleção contra os indivíduos oriundos de sementes endogâmicas.

Usualmente, a estimativa das taxas de cruzamento de populações e, conseqüentemente, de espécies é obtida a partir dos dados de progênies por representarem a fase de vida da planta mais próxima dos zigotos, o que faz com que haja uma investigação desse parâmetro sem os efeitos das possíveis pressões seletivas que as populações estariam sofrendo ao longo de seu ciclo de vida. Com isso, assumese que nessa fase do ciclo de vida das espécies não haveria seleção ou que essa seria mínima. Porém, estudos teóricos (Fisher 1941, Jain \& Workman 1967) têm mostrado que o surgimento de um gene na população, que permita a ocorrência de autofertilidade, apresenta uma vantagem adaptativa imediata. A menos que essa vantagem seja contraposta por seleção favorável aos heterozigotos, esperase que a população evolua para a autopolinização. Dessa forma, as diferenças encontradas entre as estimativas das taxas de cruzamento para as três fases do ciclo de vida de $C$. moschata (progênies, juvenis e adultos) poderiam estar relacionadas à seleção favorável aos heterozigotos. Isso corrobora o fato da população de progênies do PECB ter apresentado um déficit significativo de heterozigotos, enquanto as demais de juvenis e de adultos apresentaram uma heterozigosidade média dentro do esperado pelas proporções de Hardy-Weinberg (Moraes et al. 2002). Assim sendo, tem-se que a fase de plântulas (progênies) é indicativa do potencial genético que a população poderá vir a ser (excluindo-se os efeitos de seleção e deriva), porém, é na fase de indivíduos adultos, reprodutiva e efetivamente estabelecidos, que realmente se manifestariam os parâmetros reprodutivos e genéticos reais (P.S. Martins, comunicação pessoal).

Através do procedimento multilocos, o valor de $\hat{F}$ para as árvores maternas, pelo método NR, foi significativamente negativo $(-0,266)$, indicando que a proporção de genótipos maternos heterozigotos é maior que o esperado sob cruzamentos aleatórios. Adicionalmente, este valor -0,266 foi significativamente menor que o $\hat{F}$ obtido a partir das progênies $(0,057)$. Esses resultados indicam que pode haver uma considerável seleção contra as progênies oriundas de autofecundação em C. moschata, entre o tempo de dispersão dos diásporos e a maturação reprodutiva dos indivíduos recrutados à fase adulta, como indicado por Murawski et al. (1994).

Na tabela 1, as estimativas das freqüências alélicas atribuídas a pólen e óvulo de 35 famílias de $C$. moschata são apresentadas, bem como os testes $\chi^{2}$ de heterogeneidades das duas freqüências contribuindo ao conjunto gênico de sementes, e as estimativas dos parâmetros reprodutivos $F$, $t_{\mathrm{m}}, t_{\mathrm{s}}, r_{\mathrm{s}} \mathrm{e} r_{\mathrm{p}}$. O $\chi^{2}$ de aderência ao modelo foi significativo para 11 dos 16 locos polimórficos analizados em C. moschata. Um dos fatores preponderantes para a ocorrência de $\chi^{2}$ significativos, para o teste de adequação ao modelo de cruzamento misto, são os cruzamentos preferenciais (Ritland \& El-Kassaby 1985). Os locos significativos foram eliminados e o programa foi rodado novamente, sendo que nem a taxa de cruzamento, nem os demais parâmetros foram notavelmente alterados. Da mesma forma, apenas 12 das 35 famílias analizadas mostraram-se satisfatórias, com as demais apresentando valores $\hat{t} \mathrm{~m}$ convergindo para 2,0 através do método NR. Isto deve-se à falha do modelo ao deparar-se com múltiplos genótipos maternos heterozigotos, não os discriminando de cruzamentos não preferenciais per se (Cheliak et al. 1983, Brown et al. 1985, Boshier et al. 1995). Com isto, no presente trabalho, são apresentados os resultados para o conjunto completo dos dados, que são discutidos a partir daqueles obtidos pelo método EM, que assegurou a convergência e é o mais indicado para as peculiaridades de violações dos pressupostos do modelo, apresentadas pelos dados analisados. Pelos dois métodos de maximização da equação de verossimilhança, EM e NR, a população mostrou-se com um sistema reprodutivo predominantemente alogâmico, com taxas de cruzamento multilocos $\left(\hat{t}_{\mathrm{m}}\right)$ de $0,884 \pm 0,034$ e $0,892 \pm 0,034$, respectivamente. Esses dois métodos não deveriam dar estimativas diferentes dos parâmetros. Porém, como o valor máximo de $\hat{F}$ não foi superior a 0,1 , o método EM truncou o valor de $\hat{F}$ a zero. Quaisquer diferenças entre esses métodos são devidas à natureza aproximativa dos dois procedimentos de iteração numérica, pois há sempre uma pequena aleatoriedade em onde terminam as iterações (K. Ritland, comunicação pessoal).

A diferença significativa encontrada entre $\hat{t}_{\mathrm{m}}(0,884)$

e $\hat{t}_{\text {eq }}(0,51)$ pode ser devida a uma outra fonte de endogamia (p. ex., cruzamentos entre aparentados independentes da distância entre os indivíduos) além da autopolinização (Pérez-Nasser et al. 1993). Adicionalmente, trabalhos com simulações em computador têm mostrado que as estimativas da taxa de cruzamento aparente apresentam um viés negativo, produzindo subestimativas do valor real da taxa de cruzamento (M.S. Reis, dados não publicados).

As freqüências de pólen e de óvulos diferiram significativamente umas das outras para todos os locos, com exceção de Alp-5, Cat-2, Cat-3, Cat-4, e Per-1. Essa violação da pressuposição de equivalência de freqüências alélicas de pólen recebido pelas árvores maternas tem um efeito não mensurável, mas relativamente pequeno sobre a 
Moraes, P. L. R. e Monteiro, R. - Biota Neotropica, v2 (n2) - BN01102022002

\begin{tabular}{|c|c|c|c|c|c|c|}
\hline Loco & $N$ & Alelo & Pólen (EP) & Ovulo (EP) & $\hat{F}_{\mathrm{ST}}$ & $\chi^{2}$ \\
\hline Alp-1 & 692 & $\begin{array}{l}1 \\
2\end{array}$ & $\begin{array}{l}0,247(0,047) \\
0,753(0,047)\end{array}$ & $\begin{array}{l}0,343(0,038) \\
0,657(0,038)\end{array}$ & 0,011 & $7,666 * *$ \\
\hline Alp-3 & 692 & $\begin{array}{l}1 \\
2\end{array}$ & $\begin{array}{l}0,875(0,056) \\
0,125(0,056)\end{array}$ & $\begin{array}{l}0,714(0,051) \\
0,286(0,051)\end{array}$ & 0,039 & $27,472 * * *$ \\
\hline Alp-4 & 692 & $\begin{array}{l}1 \\
2\end{array}$ & $\begin{array}{l}0,998(0,000) \\
0,002(0,000)\end{array}$ & $\begin{array}{l}0,871(0,037) \\
0,129(0,037)\end{array}$ & 0,066 & $45,586 * * *$ \\
\hline Alp-5 & 692 & $\begin{array}{l}1 \\
2\end{array}$ & $\begin{array}{l}0,002(0,000) \\
0,998(0,000)\end{array}$ & $\begin{array}{l}0,014(0,000) \\
0,986(0,000)\end{array}$ & 0,004 & 3,114 \\
\hline Cat-1 & 413 & $\begin{array}{l}1 \\
2\end{array}$ & $\begin{array}{l}0,296(0,091) \\
0,704(0,091)\end{array}$ & $\begin{array}{l}0,514(0,050) \\
0,486(0,050)\end{array}$ & 0,049 & $20,362 * * *$ \\
\hline Cat-2 & 668 & $\begin{array}{l}1 \\
2\end{array}$ & $\begin{array}{l}0,652(0,072) \\
0,348(0,072)\end{array}$ & $\begin{array}{l}0,657(0,056) \\
0,343(0,056)\end{array}$ & 0,000027 & 0,018 \\
\hline Cat-3 & 692 & $\begin{array}{l}1 \\
2\end{array}$ & $\begin{array}{l}0,452(0,074) \\
0,548(0,074)\end{array}$ & $\begin{array}{l}0,400(0,041) \\
0,600(0,041)\end{array}$ & 0,003 & 1,913 \\
\hline Cat-4 & 692 & $\begin{array}{l}1 \\
2\end{array}$ & $\begin{array}{l}0,860(0,031) \\
0,140(0,031)\end{array}$ & $\begin{array}{l}0,886(0,037) \\
0,114(0,037)\end{array}$ & 0,001 & 1,055 \\
\hline Got-1 & 692 & $\begin{array}{l}1 \\
2 \\
3\end{array}$ & $\begin{array}{l}0,798(0,025) \\
0,068(0,016) \\
0,135(0,027)\end{array}$ & $\begin{array}{l}0,657(0,053) \\
0,086(0,037) \\
0,257(0,045)\end{array}$ & 0,020 & $28,439 * * *$ \\
\hline Got-2 & 692 & $\begin{array}{l}1 \\
2 \\
3\end{array}$ & $\begin{array}{l}0,381(0,037) \\
0,229(0,036) \\
0,390(0,038)\end{array}$ & $\begin{array}{l}0,414(0,042) \\
0,129(0,046) \\
0,457(0,054)\end{array}$ & 0,006 & $8,547 *$ \\
\hline Per-1 & 679 & $\begin{array}{l}1 \\
2\end{array}$ & $\begin{array}{l}0,597(0,086) \\
0,403(0,086)\end{array}$ & $\begin{array}{l}0,643(0,067) \\
0,357(0,067)\end{array}$ & 0,002 & 1,524 \\
\hline Per-2 & 692 & $\begin{array}{l}1 \\
2\end{array}$ & $\begin{array}{l}0,455(0,090) \\
0,545(0,090)\end{array}$ & $\begin{array}{l}0,686(0,048) \\
0,314(0,048)\end{array}$ & 0,054 & $37,675 * * *$ \\
\hline Per-3 & 692 & $\begin{array}{l}1 \\
2\end{array}$ & $\begin{array}{l}0,795(0,070) \\
0,205(0,070)\end{array}$ & $\begin{array}{l}0,643(0,053) \\
0,357(0,053)\end{array}$ & 0,028 & $19,783 * * *$ \\
\hline Per-4 & 692 & $\begin{array}{l}1 \\
2 \\
3\end{array}$ & $\begin{array}{l}0,068(0,019) \\
0,765(0,042) \\
0,167(0,036)\end{array}$ & $\begin{array}{l}0,100(0,034) \\
0,671(0,047) \\
0,229(0,043)\end{array}$ & 0,008 & $10,820 * *$ \\
\hline Per-5 & 692 & $\begin{array}{l}1 \\
2\end{array}$ & $\begin{array}{l}0,282(0,043) \\
0,718(0,043)\end{array}$ & $\begin{array}{l}0,414(0,032) \\
0,586(0,032)\end{array}$ & 0,019 & $13,285 * * *$ \\
\hline Ppo-4 & 692 & $\begin{array}{l}1 \\
2\end{array}$ & $\begin{array}{l}0,558(0,068) \\
0,442(0,068)\end{array}$ & $\begin{array}{l}0,343(0,039) \\
0,657(0,039)\end{array}$ & 0,047 & $32,304 * * *$ \\
\hline & & & EM & NR & & \\
\hline $\begin{array}{l}\hat{F} \\
\hat{t}_{\mathrm{m}} \\
\hat{t}_{\mathrm{S}} \\
\hat{t}_{\mathrm{m}}-\hat{t}_{\mathrm{S}} \\
\hat{r}_{\mathrm{S}} \\
\hat{r}_{\mathrm{p}}\end{array}$ & & & $\begin{array}{l}0,000(0,000) \\
0,884(0,034) \\
0,725(0,041) \\
0,159(0,027) \\
0,357(0,063) \\
0,990(0,015)\end{array}$ & $\begin{array}{r}-0,266(0,069) \\
0,892(0,034) \\
0,686(0,048) \\
0,206(0,032) \\
0,641(0,118) \\
0,990(0,006)\end{array}$ & & \\
\hline
\end{tabular}

$\hat{F}=$ coeficiente médio de endogamia uniloco (variância mínima) para as mães; $\hat{t}_{\mathrm{m}}=$ taxa de cruzamento multilocos populacional; $\hat{t}_{\mathrm{s}}=$ taxa média de cruzamento unilocos populacional (variância mínima); $\hat{r}_{\mathrm{s}}=$ correlação entre duas progênies irmãs oriundas de autofecundação (variação normalizada da taxa de cruzamento entre as progênies); $\hat{r}_{\mathrm{p}}=$ correlação entre duas progênies irmãs oriundas de paternidade por exocruzamento (probabilidade de que um par de progênies escolhidas ao acaso de uma mesma família são irmãs-germanas). EM = método "Expectation-Maximization"; NR = método "Newton-Raphson" de maximização da equação de verossimilhança. *p<0,05; **p<0,01; ***p $<0,001$. Testes $\chi^{2}$ para heterogeneidade onde $\chi^{2}=N \hat{F}_{\mathrm{ST}}(k-1)$ com $k-1$ graus de liberdade, onde $\hat{F}_{\mathrm{ST}}$ é a medida de diversidade genética entre os conjuntos de pólen e óvulo, $N$ é o número de gametas nos dois grupos e $k$ é o número de alelos.

Tabela 1: Estimativas das freqüências alélicas atribuídas a pólen e óvulo, calculadas separadamente, de 35 famílias de Cryptocarya moschata Nees, e testes de $\chi^{2}$ de heterogeneidades das duas freqüências contribuindo ao conjunto gênico de plântulas. Estimativas dos parâmetros reprodutivos $\mathrm{F}, \mathrm{t}_{\mathrm{m}}, \mathrm{t}_{\mathrm{s}}, \mathrm{r}_{\mathrm{s}} \mathrm{e} \mathrm{r}_{\mathrm{p}}$. Erro padrão entre parênteses; $\mathrm{N}=$ número de indivíduos. 
estimativa da taxa de cruzamento populacional verdadeira (Ritland \& Jain 1981). Essa heterogeneidade encontrada pode ser causada por fatores como estrutura familiar, padrões de florescimento diferenciado entre os indivíduos, ou qualquer fator que cause variação no comportamento dos polinizadores nas diferentes árvores, tais como mudança na produção de néctar, grau de isolamento dos indivíduos, diferenças no tempo de florescimento dos indivíduos, diferença funcional da flor, como macho ou fêmea, entre os indivíduos, pólen imigrante de fora da população amostrada, ou a partir de uma amostra não representativa das árvores maternas da população (Murawski \& Hamrick 1991, 1992b). Essa última alternativa enquadra-se no presente estudo, uma vez que foram analisadas 35 das 141 árvores de $C$. moschata amostradas na população, sendo que a densidade estimada foi de 12 indivíduos reprodutivos por hectare (Moraes et al. 1999). Esses resultados são indicadores, também, de padrões de cruzamento não aleatório dentro da população estudada do PECB.

A diferença entre $\hat{t}_{\mathrm{m}}$ e $\hat{t}_{\mathrm{S}}$ foi significativa, indicando haver endogamia biparental em torno de $16 \%$. Devido às possíveis violações do modelo de cruzamento misto (Brown et al. 1985), a estimativa mais realista da taxa de cruzamento é a de multilocos, por ser mais robusta e precisa (Ritland 1990). Contudo, as simulações feitas por Ritland \& Jain (1981) mostraram a permanência de estimativas viesadas de autofecundação quando poucos locos foram utilizados, sugerindo que um método mais direto torna-se necessário para se estimar a proporção de endogamia causada por cruzamentos endogâmicos. Um método dessa natureza seriam as estimativas multilocos conjuntas de $t$ e $p$ para as árvores individuais (Ritland \& El-Kassaby 1985), apresentado na tabela 2 .

Há dois procedimentos para se obter estas estimativas de cada árvore: 1 ) estimando-se apenas $t$, mantendo-se $p$ constante ao nível populacional; 2) estimando-se $t$ e $p$ conjuntamente. A estimativa média de $t$ usando o primeiro procedimento foi 0,878 , enquanto que pelo segundo foi 0,973 , relativamente maior do que a encontrada para a população.

As estimativas multilocos conjuntas de $t$ e $p$ tendem produzir: 1) estimativas de $t$ que excluem endogamia efetiva causada por cruzamento entre parentes mais distantes; e 2) estimativas de $p$ que incluem pólen de cruzamentos endogâmicos (p.ex., pólen relacionado à planta mãe, Ritland $\&$ Ganders 1985). Porém, apenas o componente feminino do cruzamento foi medido. Quando apenas o valor de $t$ foi medido para cada árvore, a estimativa média para as árvores permaneceu semelhante à obtida para a população, provavelmente pelas estimativas de $t$ ainda incluirem uma "endogamia efetiva" (termo designado por Ritland [1984] para incluir tanto a autofecundação, como o cruzamento entre os indivíduos aparentados) causada pela covariância

\begin{tabular}{|c|c|c|c|}
\hline & \multirow{2}{*}{$\mathrm{N}$} & \multicolumn{2}{|l|}{$\mathrm{a}$} \\
\hline & & $\hat{t}_{\mathrm{m}}( \pm \mathrm{SE})$ & $\hat{t}_{\mathrm{m}}( \pm \mathrm{SE})$ \\
\hline $\begin{array}{l}\text { População } \\
\text { Árvores }\end{array}$ & 692 & $0,893(0,033)$ & $0,885(0,035)$ \\
\hline 091 & 20 & $1,00(0,00)$ & $1,00(0,00)$ \\
\hline 136 & 20 & $0,51(0,11)$ & $0,65(0,19)$ \\
\hline 138 & 20 & $0,63(0,11)$ & $0,95(0,05)$ \\
\hline 139 & 20 & $1,00(0,03)$ & $1,00(0,00)$ \\
\hline 156 & 20 & $0,72(0,14)$ & $1,00(0,00)$ \\
\hline 164 & 20 & $0,88(0,09)$ & $1,00(0,00)$ \\
\hline 167 & 20 & $0,85(0,12)$ & $1,00(0,00)$ \\
\hline 230 & 20 & $0,95(0,07)$ & $1,00(0,00)$ \\
\hline 232 & 20 & $0,82(0,13)$ & $1,00(0,00)$ \\
\hline 237 & 20 & $0,90(0,00)$ & $0,90(0,00)$ \\
\hline 239 & 20 & $0,92(0,06)$ & $1,00(0,00)$ \\
\hline 240 & 20 & $0,59(0,14)$ & $1,00(0,00)$ \\
\hline 241 & 20 & $1,00(0,00)$ & $1,00(0,00)$ \\
\hline 243 & 20 & $1,00(0,00)$ & $1,00(0,00)$ \\
\hline 244 & 20 & $0,90(0,00)$ & $0,90(0,00)$ \\
\hline 247 & 20 & $0,99(0,00)$ & $0,99(0,00)$ \\
\hline 252 & 20 & $0,99(0,00)$ & $0,99(0,00)$ \\
\hline 256 & 20 & $1,00(0,00)$ & $1,00(0,00)$ \\
\hline 258 & 20 & $0,89(0,08)$ & $1,00(0,00)$ \\
\hline 260 & 20 & $0,27(0,12)$ & $1,00(0,00)$ \\
\hline 261 & 20 & $0,82(0,09)$ & $0,95(0,06)$ \\
\hline 263 & 20 & $1,00(0,00)$ & $1,00(0,00)$ \\
\hline 270 & 20 & $0,57(0,12)$ & $0,84(0,14)$ \\
\hline 275 & 20 & $1,00(0,00)$ & $1,00(0,00)$ \\
\hline 295 & 16 & $1,00(0,00)$ & $1,00(0,00)$ \\
\hline 296 & 20 & $1,00(0,00)$ & $1,00(0,00)$ \\
\hline 297 & 20 & $0,90(0,00)$ & $0,90(0,00)$ \\
\hline 298 & 20 & $1,00(0,00)$ & $1,00(0,00)$ \\
\hline 309 & 20 & $0,78(0,14)$ & $1,00(0,00)$ \\
\hline 310 & 20 & $1,00(0,00)$ & $1,00(0,00)$ \\
\hline 313 & 20 & $1,00(0,00)$ & $1,00(0,00)$ \\
\hline 318 & 20 & $0,97(0,05)$ & $1,00(0,00)$ \\
\hline 319 & 20 & $1,00(0,00)$ & $1,00(0,00)$ \\
\hline 320 & 16 & $1,00(0,00)$ & $1,00(0,00)$ \\
\hline 321 & 20 & $0,88(0,09)$ & $1,00(0,00)$ \\
\hline MÉDIA & & 0,878 & 0,973 \\
\hline
\end{tabular}

${ }^{a}=$ cálculos de $\hat{t}_{\mathrm{m}}$ para árvores individuais com as frequiências gênicas de pólen mantidas constantes ao nível populacional; ${ }^{\mathrm{b}}=$ cálculos de $\hat{t}_{\mathrm{m}}$ para árvores individuais com os valores de $\hat{t}$ e $\hat{p}$ estimados conjuntamente; erros padrões obtidos a partir de 1.000 bootstraps.

Tabela 2: Taxas de cruzamento multilocos populacional e de árvores individuais de Cryptocarya moschata Nees amostradas no $P E C B$.

das frequiências alélicas de pólen com o genótipo materno. Por outro lado, quando $t$ e $p$ foram estimados conjuntamente para as árvores individuais, a estimativa média foi de 0,973 , indicando ausência quase total de endogamia. Isso sugere que a diferença entre a estimativa populacional e a média de árvores individuais é causada por cruzamentos endogâmicos.

A correlação entre dois irmãos de paternidade por exocruzamento foi em torno de $99 \%$. Como estas progênies são oriundas de flores polinizadas em diferentes dias, essa correlação é provável de ser originada de cruzamentos de um pequeno conjunto de vizinhos próximos. Se houver $n$ vizinhos próximos e cada um tiver a mesma probabilidade de ser o parental masculino, com os cruzamentos 
consecutivos sendo independentes (envolvendo visitas separadas dos polinizadores), então a probabilidade de um mesmo vizinho se acasalar duas vezes é $1 / n$ (Ritland 1989). Dessa forma, para C. moschata, o cruzamento aleatório com um único vizinho próximo poderia resultar nessa correlação observada de $99 \%$ de irmãos germanos. Por outro lado, cerca de $35,7 \%$ da endogamia efetiva encontrada pode ser explicada pela autopolinização das árvores, como indicado por $\hat{r}_{\mathrm{s}}$.

Como apontado por Ritland (1989), pela adoção de pares de irmãos como unidade observacional, dois componentes adicionais do sistema reprodutivo podem ser identificados: a correlação de autofecundação e a correlação de paternidade por exocruzamento, sendo ambas as correlações necessárias para a determinação das correlações genéticas dos mesmos. Em contraposição, se a preocupação for apenas com a modificação geradora da homozigosidade populacional, a taxa de cruzamento por si só é suficiente para a descrição da transmissão genética na população. Essas correlações servem como indicadores da variação nas freqüências de pólen recebido por cada planta, e da variação na taxa de autocruzamento. Isso é esperado ocorrer em populações naturais, onde as árvores diferem em seus períodos de florescimento, grau de isolamento e composição de espécies, bem como no comportamento dos polinizadores que as visitam (Rocha \& Lobo 1998).

O valor encontrado da taxa de cruzamento multilocos para a população de C. moschata do PECB, 0,884, é comparável às estimativas registradas de populações de espécies arbóreas neotropicais (O’Malley \& Bawa 1987, O’Malley et al. 1988, Murawski \& Hamrick 1991, 1992b, Murawski et al. 1994). O fato de espécies como C. moschata apresentarem um pequeno grau de endogamia em populações naturais manifesta a questão de quais mecanismos favoreceriam esse fenômeno e preveniriam a evolução de um sistema reprodutivo predominantemente autógamo, como apontado por Brown et al. (1975).

\section{Agradecimentos}

Ao Prof. Paulo Sodero Martins (in memoriam) pelo inestimável apoio; ao Prof. Roland Vencovsky, pelas sugestões oferecidas ao manuscrito; ao Instituto Florestal de São Paulo pela permissão de coleta de material.

\section{Referências bibliográficas}

ALFENAS, A.C., PETERS, I., BRUNE, W. \& PASSADOR, G.C. 1991. Eletroforese de proteínas e isoenzimas de fungos e essências florestais. Universidade Federal de Viçosa, Viçosa.

ALLARD, R.W. \& WORKMAN, P.L. 1963. Population studies in predominantly self-pollinated species. IV.
Seasonal fluctuations in estimated values of genetic parameters in lima bean populations. Evolution 17:470480.

BAWA, K.S. 1974. Breeding systems of tree species of a lowland tropical community. Evolution 28:85-92.

BAWA, K.S. 1976. Breeding of tropical hardwoods: an evaluation of underlying bases, current status and future prospects. In Tropical trees: variation, breeding and conservation (J. Burley \& B.T. Styles, eds.). Academic Press, London, p.43-59.

BAWA, K.S., PERRY, D.R. \& BEACH, J.H. 1985. Reproductive biology of tropical lowland rain forest trees. 1. Sexual systems and incompatibility mechanisms. Am. J. Bot. 72:331-345.

BOSHIER, D.H., CHASE, M.R. \& BAWA, K.S. 1995. Population genetics of Cordia alliodora (Boraginaceae), a neotropical tree. 2. Mating system. Am. J. Bot. 82:476483.

BROWN, A.H.D. 1989. Genetic characterization of plant mating systems. In Plant population genetics, breeding and germplasm resources (A.H.D. Brown, M.T. Clegg, A.L. Kahler \& B.S. Weir, eds.). Sinauer Associates, Sunderland, p.145-162.

BROWN, A.H.D. \& ALLARD, R.W. 1970. Estimation of the mating system in open-pollinated maize populations using isozyme polymorphisms. Genetics 66:133-145.

BROWN, A.H.D., BARRETT, S.C.H. \& MORAN, G.F. 1985. Mating system estimation in forest trees: models, methods and meanings. In Population genetics in forestry (H.R. Gregorius, ed.). Springer-Verlag, Berlin, p.32-49.

BROWN, A.H.D., MATHESON, A.C. \& ELDRIDGE, K.G. 1975. Estimation of the mating system of Eucalyptus obliqua L'Herit. by using allozyme polymorphisms. Austr. J. Bot. 23:931-949.

BULLOCK, S.H. 1985. Breeding systems in the flora of a tropical deciduous forest in Mexico. Biotropica 17:287301.

CHELIAK, W.M., STROBECK, K.M.C., YEH, F.C.H. \& DANCIK, B.P. 1983. Estimation of mating system parameters in plant populations using EM algorithm. Theor. Appl. Genet. 65:157-161.

CLEGG, M.T. 1980. Measuring plant mating systems. Bioscience 30:814-818.

EL-KASSABY, Y.A. \& RITLAND, K. 1987. Low levels of pollen contamination in a Douglas-fir seed orchard as detected by allozyme markers. Silvae Genetica 35:224229.

FISHER, R.A. 1941. Average excess and average effect of a gene substitution. Ann. Eugen. 11:53-63.

HALL, P., ORRELL, L.C. \& BAWA, K.S. 1994. Genetic diversity and mating system in a tropical tree, Carapa 
guianensis (Meliaceae). Am. J. Bot. 81:1104-1111.

HAMRICK, J.L. 1990. Isozymes and the analysis of genetic structure in plant populations. In Isozymes in plant biology (D.E. Soltis \& P.S. Soltis, eds.). Chapman \& Hall, London, p.87-105.

HAMRICK, J.L. \& GODT, M.J. 1990. Allozyme diversity in plant species. In Population genetics and germplasm resources in crop improvement (A.H.D. Brown, M.T. Clegg, A.L. Kahler \& B.S. Weir, eds.). Sinauer, Sunderland, MA, p. 43-63.

HAMRICK, J.L. \& SCHNABEL, A. 1985. Understanding the genetic structure of plant populations: some old problems and a new approach. In Population genetics in forestry (H.R. Gregorius, ed.). Springer-Verlag, Berlin, p.50-70.

JAIN, S.K. \& WORKMAN, P.L. 1967. Generalized $F$-statistics and the theory of inbreeding and selection. Nature 214:674-678.

KAGEYAMA, P.Y. 1990. Genetic structure of tropical tree species of Brazil. In Reproductive ecology of tropical forest plants (K.S. Bawa \& M. Hadley, eds.). UNESCO / The Parthenon Publishing Group, Paris, p.375-387.

KEPHART, S.R. 1990. Starch gel electrophoresis of plant isozymes: a comparative analysis of techniques. Am. J. Bot. 77:693-712.

LOVELESS, M.D. \& HAMRICK, J.L. 1984. Ecological determinants of genetic structure in plant populations. Ann. Rev. Ecol. Syst. 15:65-95.

MORAN, G.F. \& BROWN, A.H.D. 1980. Temporal heterogeneity of outcrossing rates in alpine ash (Eucalyptus delegatensis R. T. Bak.). Theor. Appl. Genet. 57:101-105.

MORAES, P.L.R., MONTEIRO, R. \& VENCOVSKY, R. 1999. Conservação genética de populações de Cryptocarya moschata Nees (Lauraceae) na Mata Atlântica do estado de São Paulo. Rev. Bras. Bot. 22:237-248. (http://

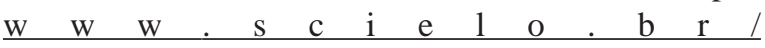
scielo.php?script $=$ sci_abstract\&pid=S010084041999000500004\&lng=en\&nrm=iso)

MORAES, P.L.R., MONTEIRO, R. \& VENCOVSKY, R. 2002. Genetic differentiation and diversity of natural populations of Cryptocarya spp. (Lauraceae) from the Brazilian Atlantic rain forest. Lundiana 3(2) (no prelo).

MURAWSKI, D.A. \& BAWA, K.S. 1994. Genetic structure and mating system of Stemonoporus oblongifolius (Dipterocarpaceae) in Sri Lanka. Am. J. Bot. 81:155-160.

MURAWSKI, D.A. \& HAMRICK, J.L. 1991. The effect of the density of flowering individuals on the mating systems of nine tropical tree species. Heredity 67:167174.

MURAWSKI, D.A. \& HAMRICK, J.L. 1992a. Mating system and phenology of Ceiba pentandra (Bombacaceae) in
Central Panama. J. Hered. 83:401-404.

MURAWSKI, D.A. \& HAMRICK, J.L. 1992b. The mating system of Cavanillesia platanifolia under extremes of flowering-tree density: a test of predictions. Biotropica 24:102-104.

MURAWSKI, D.A., DAYANANDAN, B. \& BAWA, K.S. 1994. Outcrossing rates of two endemic Shorea species from Sri Lankan tropical rain forests. Biotropica 26:2329.

MURAWSKI, D.A., HAMRICK, J.L., HUBBELL, S.P. \& FOSTER, R.B. 1990. Mating systems of two bombacaceous trees of a neotropical moist forest. Oecologia 82:501-506.

NEI, M. \& SYAKUDO, K. 1958. The estimation of outcrossing in natural populations. Japan. J. Genetics $33: 46-51$

O’MALLEY, D.M. \& BAWA, K.S. 1987. Mating system of a tropical rain forest tree species. Am. J. Bot. 74:1143-1149.

O'MALLEY,D.M., BUCKLEY,D.P., PRANCE, G.T. \& BAWA, K.S. 1988. Genetics of Brazil nut (Bertholletia excelsa Humb. \& Bonpl.: Lecythidaceae). 2. Mating system. Theor. Appl. Genet. 76:929-932.

PAIVA, J.R., KAGEYAMA, P.Y. \& VENCOVSKY, R. 1993. Outcrossing rates and inbreeding coefficients in rubber trees (Hevea brasiliensis (Willd. ex Adr. de Juss.) Müeller Arg.). Rev. Bras. Genética 16:1003-1011.

PAIVA, J.R., KAGEYAMA, P.Y. \& VENCOVSKY, R. 1994. Genetics of rubber tree (Hevea brasiliensis (Willd. ex Adr. de Juss.) Müll. Arg.) 2. Mating system. Silvae Genetica 43:373-376.

PÉREZ-NASSER, N., EGUIARTE, L.E. \& PIÑERO, D. 1993. Mating system and genetic structure of the distylous tropical tree Psychotria faxlucens (Rubiaceae). Am. J. Bot. 80:45-52.

RITLAND, K. 1984. The effective proportion of selffertilization with consanguineous matings in inbred populations. Genetics 106:139-152.

RITLAND, K. 1989. Correlated matings in the partial selfer Mimulus guttatus. Evolution 43:848-859.

RITLAND, K. 1990. A series of FORTRAN computer programs for estimating plant mating systems. J. Hered. $81: 235-237$

RITLAND, K. \& EL-KASSABY, Y.A. 1985. The nature of inbreeding in a seed orchard of Douglas fir as shown by an efficient multilocus model. Theor. Appl. Genet. 71:374384.

RITLAND, K. \& GANDERS, F.R. 1985. Variation in the mating system of Bidens menziesii (Asteraceae) in relation to population substructure. Heredity 55:235-244.

RITLAND, K. \& JAIN, S. 1981. A model for the estimation 
of outcrossing rate and gene frequencies using $n$ independent loci. Heredity 47:35-52.

ROCHA, O.J. \& LOBO, J.A. 1998. Genetic diversity and outcrossing rates in the guanacaste tree (Enterolobium cyclocarpum Jacq.) in the dry forests of Costa Rica. In Recent Advances in Biotechnology for Tree Conservation and Management, Proceedings of an IFS Workshop. International Foundation for Science (IFS), Stockholm, p.65-81.

SHAW, D.V. \& ALLARD, R.W. 1981. Estimation of outcrossing rates in Douglas-fir using isozyme markers. Theor. Appl. Genet. 62:113-120.

STACY, E.A., HAMRICK, J.L., NASON, J.D., HUBBELL, S.P., FOSTER, R.B. \& CONDIT, R. 1996. Pollen dispersal in low-density populations of three neotropical tree species. Am. Natur. 148:275-298.

WRIGHT, S. 1921. Systems of mating. Genetics 6:111-178.

ZAPATA, T.R. \& KALIN-ARROYO, M.T. 1978. Plant reproductive ecology of a secondary deciduous tropical forest in Venezuela. Biotropica 10:221-230.

Título: Taxas de cruzamento em uma população natural de Cryptocarya moschata Nees (Lauraceae)

Autores: Moraes, P. L. R. e Monteiro, R.

Biota Neotropica, Vol. 2 (número 2): 2002

http://www.biotaneotropica.org.br/v2n2/pt/ a bstract ?artic 1 e + B N 01102022002

Recebido em 12/04/2002 - Revisado em 09/07/2002 -

Publicado em 07/08/2002

ISSN 1676-0603

http://www.biotaneotropica.org.br 